\title{
Physics and Commissioning of the SNO+ Experiment
}

\author{
Jack Dunger* for the SNO+ Collaboration \\ University of Oxford \\ E-mail: jack.dunger@physics.ox.ac.uk
}

\begin{abstract}
A brief update on the status and future prospects of the SNO+ experiment. The aims and progress of three data taking phases are reviewed with a particular emphasis on the search for neutrinoless double beta decay in ${ }^{130} \mathrm{Te}$. A single/multi-site discrimination method based on timing distortion of scintillation pulses is presented with reference to background ${ }^{60} \mathrm{Co}$ decay. A projected limit on the effective Majorana neutrino mass of $m_{\beta \beta}<38.7 \mathrm{meV}$ at $90 \%$ confidence is compared with existing measurements and the capabilities of an upgraded SNO+ detector are discussed.
\end{abstract}

The European Physical Society Conference on High Energy Physics

5-12 July, 2017

Venice

${ }^{*}$ Speaker. 


\section{SNO+ detector}

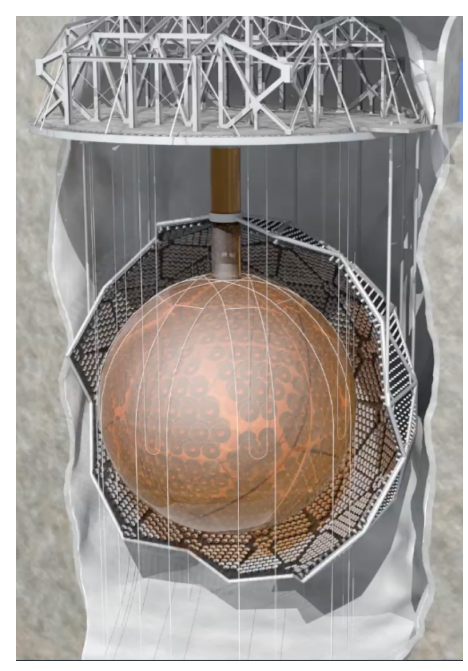

Figure 1: A cross-section of the $\mathrm{SNO}+$ detector

Located 2km underground in SNOLAB, Sudbury, Canada, the SNO+ detector is an extremely sensitive, kTon scale photon counter. Figure 1 shows a cross section of the detector. At the center of the detector a $5 \mathrm{~cm}$ thick acrylic vessel (AV) of radius $6 \mathrm{~m}$ supports a liquid target. The target is monitored by $\approx 9300$ inward facing PMTs placed $880 \mathrm{~cm}$ from the center. The volume between the AV and the PMTs and between the PMTs and the rock is filled with ultra pure water (UPW) to provide optical matching and to shield the target from external $\gamma$.

The data taking of the experiment will be divided in to three phases. In the water phase the target is UPW, in the scintillator phase this is replaced by an organic liquid scintillator, finally, in the Tellurium phase, Tellurium is loaded into the scintillator.

SNO+ has a rich physics program across these three phases capable of detecting solar, reactor, geo and supernova neutrinos. The primary goal however is the search for neutrinoless double beta decay $(0 v \beta \beta)$ of ${ }^{130} \mathrm{Te}$ in the Tellurium phase.

\section{Water Phase}

At the time of writing the water phase has begun and the detector has been taking steady physics data since April 2017. During that time an extensive calibration campaign was launched. A laser diffusing source was deployed for calibration of the PMTs' charge and time responses, as was an ${ }^{16} \mathrm{~N} \gamma$ source to calculate the detector's energy scale. New, in-situ, laser and LED optical calibration systems are being commissioned to measure the attenuation and scattering of the target and to monitor the charge and time response of the PMTs.

There are also physics goals for the water phase. The directionality of the Cerenkov light produced is ideal for constraining $\gamma$ rays from the AV, external water and PMTs. SNO+ will also set the most stringent limit on the invisible nucleon decay $n \rightarrow 3 v$ with 6 months of live-time [1]. 


\section{Transitioning to Liquid Scintillator}

As mentioned in section 1, the central UPW target of SNO+ will be replaced with a 780 ton scintillator target, made up of LAB (Linear Alykl Benzene) doped with $2 \mathrm{~g} / \mathrm{L}$ of the primary fluor PPO (2,5-Diphenyloxazole). There are several significant advantages to transitioning from a water Cerenkov detector to a liquid scintillator one. First, organic LAB is non-polar and therefore purifiable to 1000 times smaller concentrations of Uranium and Thorium chain contaminants. Second, scintillation of $\mathrm{LAB}$ produces more photons per $\mathrm{MeV}$ of deposited energy than Cerenkov light in water by as much as a factor of 50. At these light levels, the trigger threshold can be lowered to sub-MeV, and energy resolutions of a few $\% \cdot \sqrt{(\mathrm{E} / \mathrm{MeV})}$ can be achieved.

One disadvantage of liquid scintillator technology is that the emitted light is isotopic and therefore maintains no correlation with the direction of the incident particle. This means that solar backgrounds and signals cannot be statistically separated by their correlation with the solar direction. Of course in a scintillation detector both Cerenkov and scintillation mechanisms are at work, but the latter overwhelms the former in photons produced and any timing differences between the two are washed out by vertex resolution effects, light dispersion and the timing response of large area PMTs.

For these advantages and others the $\mathrm{SNO}+$ collaboration has constructed, and is currently commissioning, an underground $\mathrm{LAB}$ purification plant to produce $\mathrm{LAB}$ with Uranium/Thorium chain contamination of $10^{-17} \mathrm{~g}_{U, T h} / \mathrm{g}_{L A B}$ [1]. In March 2018, with commissioning complete, purified $\mathrm{LAB}$ will be fed into the neck of the AV replacing UPW removed by pipes at the bottom.

\section{Loading Tellurium}

SNO+ has chosen the double beta $(\beta \beta)$ emitter ${ }^{130} \mathrm{Te}$ for its search for $0 v \beta \beta$. At $34 \%{ }^{130} \mathrm{Te}$ has by far the largest natural abundance of any $\beta \beta$ emitter, after ${ }^{130} \mathrm{Xe}$ it has the second best expected ratio of $2 v \beta \beta$ to $0 v \beta \beta$ events and it is highly transparent in the wavelength region of PMT sensitivity [2].

The decay has a Q-value of $2.5 \mathrm{MeV}$, and the energy is deposited by two electrons. Crucially, the dominant natural radioactivity in this region is ${ }^{214} \mathrm{Bi}$ decay which can be suppressed by a factor of $5 \cdot 10^{5}$ by tagging the delayed coincidence with the ${ }^{214}$ Po decay that follows [1].

The Tellurium is sourced as telluric acid ( $\mathrm{TeA}$ ) and shipped underground where it can be easily purified by acid-base extraction. Underground purification will ensure the removal of cosmogenic isotopes. These isotopes are created by cosmic muon spallation at surface and their decays often have significant overlap with the $0 v \beta \beta$ energy spectrum.

Loading Tellurium into LAB is difficult for exactly the same reason that U/Th chain daughters are easy to remove - the non-polarity of the LAB. Loading will be achieved by first manufacturing an organometallic Tellurium complex Te-butanediol. This is readily soluble in LAB and very transparent. It does however quench the intrinsic light yield of the scintillator. After this effect we expect an energy scale of 500 photoelectrons/MeV.

At first Tellurium will be loaded to $0.5 \%$ by mass, equivalent to 300 kilograms of ${ }^{130} \mathrm{Te} .3 .8$ ton of TeA have already been shipped underground and the purification plant construction is underway. Loading will begin in January 2019. 


\begin{tabular}{ccc}
\hline Live-time/yr & $T_{1 / 2}^{0 v} / \mathrm{yr}$ & $m_{\beta \beta} / \mathrm{meV}$ \\
\hline 1 & $8.0 \cdot 10^{25}$ & 75.2 \\
5 & $2.0 \cdot 10^{26}$ & 39.7 \\
\hline
\end{tabular}

Table 1: Projected half-life and effective mass sensitivities at $90 \%$ confidence

\section{Sensitivity to Neutrinoless Double Beta Decay}

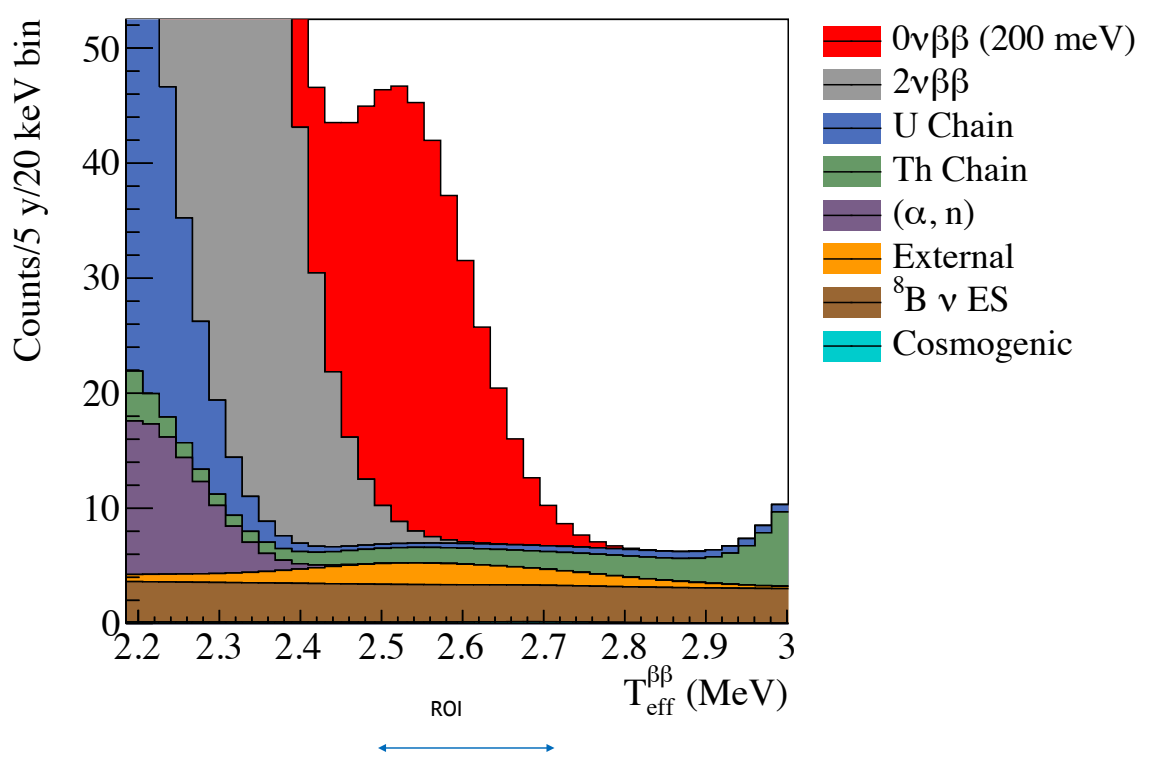

Figure 2: The expected energy spectrum near the ${ }^{130} \mathrm{Te} 2 v \beta \beta$ spectrum after 5 years live-time with a $0 v \beta \beta$ signal equivalent to a $m_{\beta \beta}=200 \mathrm{meV}$ is included.

Figure 5 shows the expected spectrum near the $2 v \beta \beta$ endpoint after 5 years live-time, including a possible signal. The detector sensitivity was assessed using a cut and count analysis with region of interest of $2.49<E / \mathrm{MeV}<2.65$ and a fiducial volume of $r / \mathrm{mm}<3500$.

In this region we expect a background of 13 counts/yr and a signal efficiency of $13 \%$, table 5 shows the expected sensitivity. The dominant backgrounds in this region are: elastic scatters from solar ${ }^{8} \mathrm{~B}$ neutrinos, $2 v \beta \beta$ decays, Uranium and Thorium decays from scintillator contamination and external $\gamma$ from radioactive decays on the acrylic vessel, PMTs and support ropes.

Figure 5 shows a comparison between the SNO+ expected limit and existing competitor experiments. It is clear that $\mathrm{SNO}+$ can set the most stringent limit on $m_{\beta \beta}$, probing well into the inverted hierarchy regime. A SNO+ phase II could achieve much greater sensitivity by increasing the loading of Tellurium and compensating for the extra light yield quenching with improved light collection. Up to three times better collection could achieved by upgrading the PMTs to modern high quantum efficiency PMTs and replacing the now aged photo-cathode concentrators. A further 


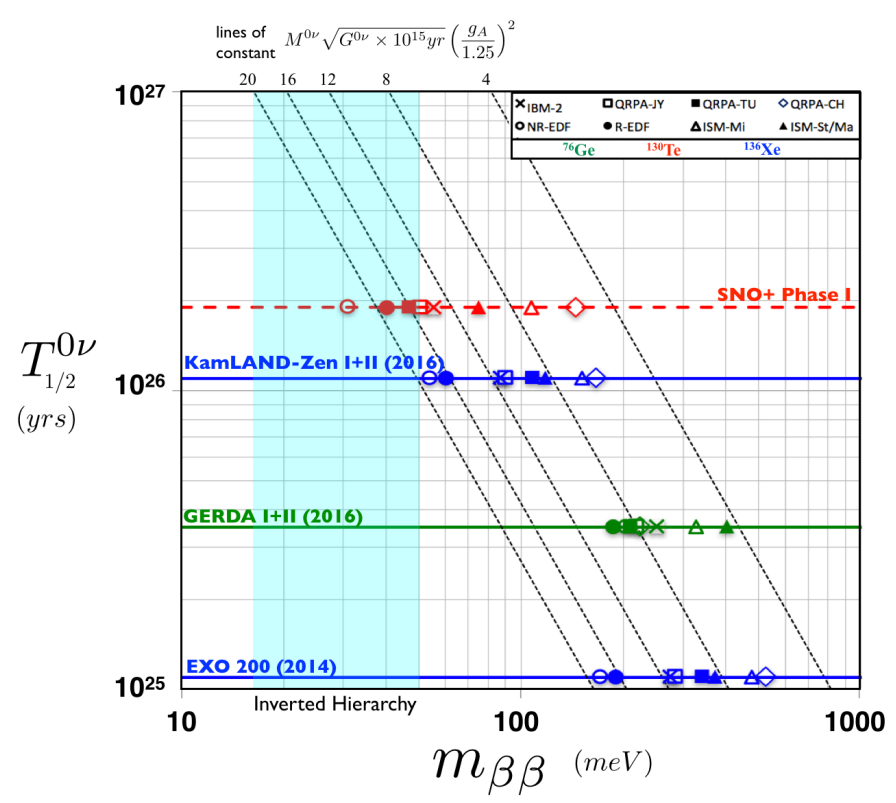

Figure 3: Comparison of $0 v \beta \beta$ experiments $m_{\beta \beta}$ sensitivity. Experiments place limits on $0 v \beta \beta$ halflives and infer limits on the effective mass $m_{\beta \beta}$. The two are related by $m_{\beta \beta} \propto T_{\frac{1}{2}}^{-\frac{1}{2}}$ but the constant of proportionality is isotope dependent and subject to theoretical uncertainties. Diagonal lines represent possible values of the constant and the points represent current estimates.

step could be to increase the photo-cathode coverage by adding extra PMTs or better tessellating concentrators. A SNO+ phase II would aim to cover the entire inverted hierarchy regime.

\section{Single/Multi-site Separation}

In the scintillator and tellurium phase, the time signature of scintillation pulses will encode information about the event topologies that produced them. Electron tracks in scintillator are significantly shorter than the expected vertex resolution and so appear point-like. Electron pulses are therefore close to the intrinsic time response of the liquid scintillator the same is true for two electron $0 v \beta \beta$ decays. On the other hand, $\gamma$ at energies $\mathscr{O}(1 \mathrm{MeV})$ free stream between multiple scatters and deposit their energy over tens of centimeters. The pulse they produce is therefore smeared out. This is observable via the time of flight corrected hit times as shown in figure 4 . The difference can be used to statistically separate single and multi-site events such as ${ }^{60} \mathrm{Co}$ decay, one cosmogenic isotope that will be removed in the underground purification plant but needs in-situ constraint. Figure 5 shows a simple linear classifier that statistically separates decays.

\section{Conclusion}

The SNO+ collaboration has begun to peruse its rich physics program by taking data with a water target. The upgrade to a liquid scintillator target in March 2018 will allow for lower 


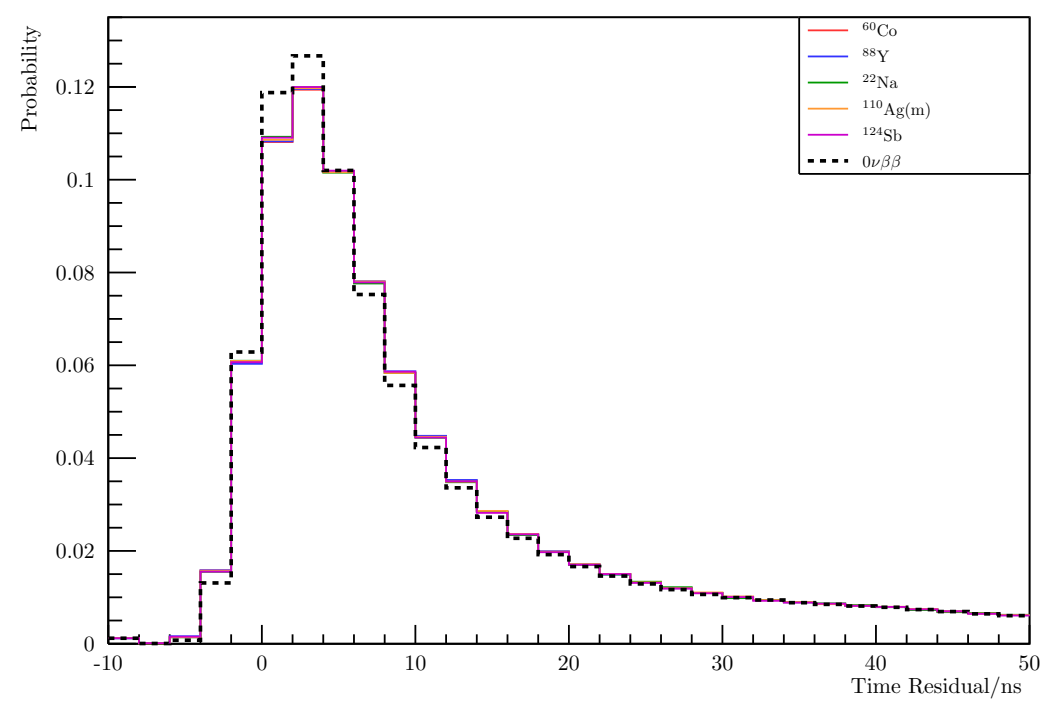

Figure 4: Simulated average time of flight corrected hit time PDFs for $0 v \beta \beta$ events and various $\beta \gamma$ decays including ${ }^{60} \mathrm{Co}$ in the Tellurium phase, $r<3500 \mathrm{~mm}$

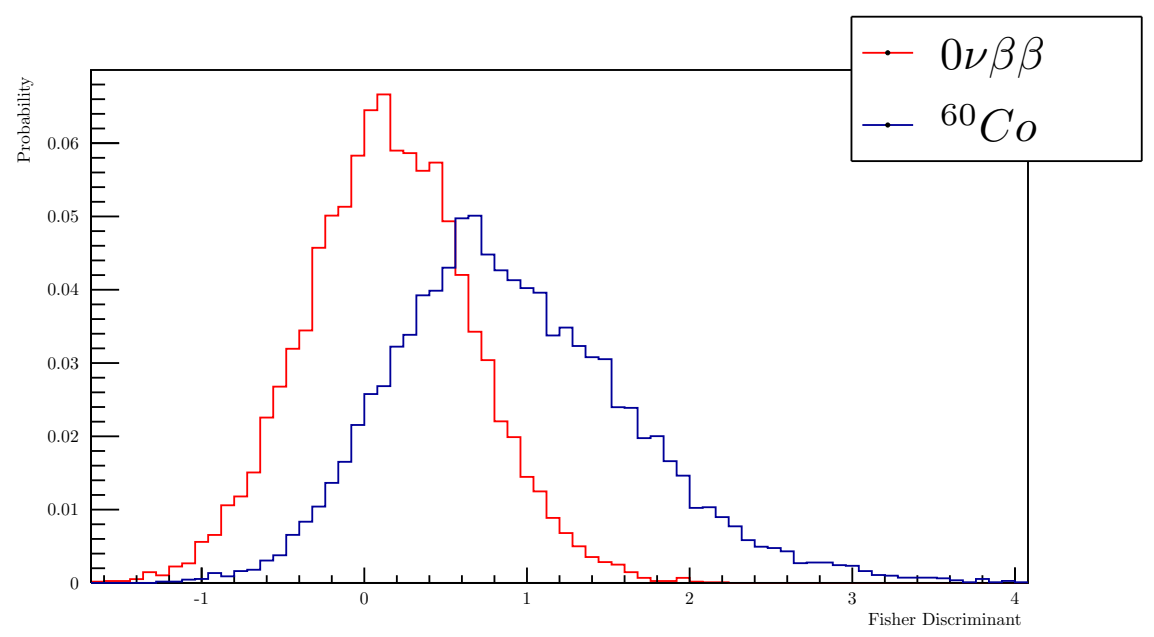

Figure 5: Fisher descriminant statistic comparing $0 v \beta \beta$ events and ${ }^{60}$ Co decays. Based on the PDFs in figure 4 and correlations betweeen bins. Simulated in the Tellurium phase, $r<3500 \mathrm{~mm}$ 
thresholds and tighter energy resolutions. After loading Tellurium into the detector in early 2019, $\mathrm{SNO}+$ will set the leading limit on $m_{\beta \beta}$ by searching for $0 v \beta \beta$ in ${ }^{130} \mathrm{Te}$. Potential $\beta \gamma$ backgrounds to this search can be constrained using the characteristic smearing of $\gamma$ ray scintillation pulses.

\section{References}

[1] SNO+ collaboration, S. Andringa et al., Current Status and Future Prospects of the SNO+ Experiment, Adv. High Energy Phys. 2016 (2016) 6194250, [1508 . 05759].

[2] S. D. Biller, Probing Majorana neutrinos in the regime of the normal mass hierarchy, Phys. Rev. D87 (2013) 071301, [1306.5654]. 